Céla Regina Gonçalves e Silva ${ }^{1}$

Karina Ester de Melo ${ }^{2}$

Mariella Vieira Pereira Leão'

Rosana Ruis ${ }^{3}$

ANTONIO OlaVo CARDOSO JORGE ${ }^{4}$

Artigos originais

Palavras-chave

Candidíase vulvovaginal Imunoglobulina A

Candida albicans

Imunidade nas mucosas

Keywords

Candidiasis, vulvovaginal Immunoglobulin A Candida albicans Immunity, mucosal

\section{Presença de Candida nas mucosas vaginal e bucal e sua relação com IgA salivar}

\author{
Relationship between Candida in vaginal and oral mucosae and \\ salivary $\operatorname{Ig} A$
}

\section{Resumo}

OBJETIVO: correlacionar a presença de leveduras do gênero Candida na cavidade bucal e vaginal de mulheres com e sem candidíase vulvovaginal (CVV) com os níveis de lgA secretora (lgAs) presentes na saliva. MÉTODOS: cinqüenta e uma mulheres foram incluídas; 13 apresentaram CV e 38 formaram o Grupo Controle. De cada paciente, foram coletados $2,0 \mathrm{~mL}$ de saliva sem estimulação e secreção vaginal com o auxílio de swab, que foi imerso a seguir em $2,0 \mathrm{~mL}$ de solução fisiológica. As amostras foram semeadas em ágar Sabouraud dextrose com cloranfenicol para isolamento e contagem de colônias, e os isolados foram identificadas fenotipicamente. Na saliva de ambos os grupos foi quantificada lgA pela técnica ELISA. RESULTADOS: nas 13 pacientes com diagnóstico clínico e micológico de CW, a média de unidades formadoras de colônias de Candida por mililitro de secreção vaginal (ufc/mL) foi de 52.723 e 23,8\% dos pacientes apresentaram colonização na mucosa bucal com menor quantidade de ufc/mL (6.030). Os níveis de lgAs na saliva foram mais baixos no grupo com CV (média de densidade: 0,3) quando comparados aos níveis de lgA do Grupo Controle (média de DO: 0,6). Onze pacientes (37\%) do Grupo Controle apresentaram colonização por Candida na cavidade bucal, com média de ufc/mL mais baixa quando comparada ao grupo com CV. O Grupo Controle também apresentou menor quantidade de ufc/mL (1.973) na cavidade vaginal quando comparado com - Grupo CVV (52.942). CONCLUSÕES: os resultados demonstraram que os pacientes com diagnóstico clínico de candidíase vulvovaginal apresentaram maior quantidade de Candida, tanto na cavidade vaginal quanto na bucal, e apresentaram menores níveis de lgA anti-Candida na saliva.

\section{Abstract}

PURPOSE: to correlate the presence of yeast from the Candida genus in the oral and vaginal cavity of women with and without vulvovaginal candidiasis (VVC), with secretor lgA levels (IgAs) present in the saliva. METHODS: among the 51 women included, 13 presented WC and 38 were the Control Group. An amount of $2.0 \mathrm{~mL}$ of saliva without stimulation was collected from each patient, plus vaginal secretion using a swab, which was then immersed in $2.0 \mathrm{~mL}$ of physiological solution. Samples were inseminated in Sabouraud dextrose agar with chloramphenicol for isolation and counting of colonies, and the isolated ones, phenotypically identified. IgA has been quantified in the saliva of the women from both groups, by the ELISA technique. RESULTS: in the 13 patients with clinical and mycological diagnosis of WC, the mean of Candida colony producing unities by milliliter of vaginal secretion (cpu/mL) was 52,723, and $23.8 \%$ of the patients presented colonization in the oral mucosa with lower amount of cup $/ \mathrm{mL}(6,030)$. The levels of IgAs in saliva were lower in the group with VVC (DO mean: 0.3), as compared to the lgA levels of the Control Group (DO mean: 0.6). Eleven patients (37\%) from the Control Group presented Candida colonization in the oral cavity, with a lower cup/mL mean, when compared to the WC Group. The Control Group also presented a lower amount of $\mathrm{cpu} / \mathrm{mL}(1,973)$ in the vaginal cavity, when compared to the WV Group $(52,942)$. CONCLUSIONS: these results have demonstrated that patients with clinical diagnosis of vaginal candidiasis presented a higher amount of Candida both in the vaginal and in the oral cavity, and presented lower levels of anti-Candida lgA in the saliva.
Correspondência:

Célia Regina Gonçalves e Silva Rodovia Álvares Lima Neto, 2.303, casa 12 - Guedes CEP 12120-000 - Tremembé/SP

Fone/Fax: (12) 3672-3402

E-mail: celiargs@ig.com.br

Recebido

$14 / 5 / 2007$
Laboratório de Microbiologia e Imunologia do Instituto Básico de Biociências da Universidade de Taubaté - UNITAU - Taubaté (SP), Brasil. 'Professor Assistente de Microbiologia e Imunologia do Instituto Básico de Biociências da Universidade de Taubaté - UNITAU Taubaté (SP), Brasil.

${ }^{2}$ Aluna do Departamento de Biologia da Universidade de Taubaté - UNITAU - Taubaté (SP), Brasil.

${ }^{3}$ Ginecologista do Hospital Universitário de Taubaté - UNITAU - Taubaté (SP), Brasil.

${ }_{4}^{4}$ Professor Titular de Microbiologia e Imunologia da Faculdade de Odontologia de São José dos Campos da Universidade Estadual Paulista - Unesp - São José dos Campos (SP), Brasil. 


\section{Introdução}

Em diferentes mucosas de indivíduos saudáveis, leveduras do gênero Candida são encontradas como parte da microbiota residente, sem desencadear processos infecciosos. De 25 a $75 \%$ dos indivíduos saudáveis podem apresentar Candida spp na mucosa bucal. Esta variação depende da amostragem populacional selecionada e da sensibilidade da metodologia utilizada para coleta e recuperação desses microrganismos ${ }^{1,2}$. Mulheres saudáveis e assintomáticas apresentam leveduras do gênero Candida na cavidade vaginal, como parte da microbiota, sendo Candida albicans a espécie prevalente ${ }^{3,4}$. Esses microrganismos são considerados oportunistas, porque em condições propícias, como presença de fatores pré-disponentes locais ou sistêmicos, podem proliferar e desencadear processos infecciosos ${ }^{2,5}$.

Candidíase vulvovaginal (CVV) é uma das principais doenças relatadas em consultórios ginecológicos. Nos Estados Unidos, a incidência de candidose vaginal dobrou na década de 1990 em relação à de 1980, ocorrendo cerca de 13 milhões de casos por ano. Infecções por outras espécies de Candida não albicans, como C. tropicalis e C. glabrata, entre outras, têm sido descritas ${ }^{6,7}$. A erradicação dessas leveduras é problemática, com recorrências freqüentes, e a espécie $C$. albicans é prevalente tanto na colonização quanto nas doenças ${ }^{8}$.

Para impedir a proliferação e progressão de candidíases localizadas e disseminadas, foram desenvolvidas defesas imunológicas humoral e celular contra esta levedura, sendo a resposta celular a mais apropriada para a erradicação de $C$. albicans. Entretanto, vários estudos demonstram a evidência de mecanismos de defesa inerentes à mucosa por tecido linfóide associado à mucosa. Dentre os mecanismos envolvidos na defesa, as imunoglobulinas da classe $\operatorname{IgA}$ presentes nas secreções e saliva desempenham papel fundamental. Nas formas de infecções por Candida, que estão limitadas à superfície epitelial, a IgA secretora (IgAs) age causando agregação de fungos e estes anticorpos específicos para C. albicans e podem inibir sua aderência às células epiteliais da mucosa, o que, conseqüentemente, impede a sua proliferação ${ }^{9-11}$.

A identificação de $\operatorname{Ig} A$ na superfície de células de $C$. albicans em pacientes com sinais clínicos de candidíase pode ser uma forte evidência de seu papel na proteção contra a doença, podendo ser utilizada, inclusive, como forma de diagnóstico ${ }^{12}$.

O objetivo do presente trabalho foi correlacionar a presença de leveduras do gênero Candida na cavidade bucal e vaginal de mulheres com e sem CVV, assim como as diferentes espécies nestes dois sítios, com os níveis de IgA presentes na saliva.

\section{Métodos}

Foram convidadas a participar do trabalho pacientes do gênero feminino que realizavam exames de rotina na Clínica de Ginecologia e Obstetrícia do Hospital Universitário de Taubaté (HUT) da Universidade de Taubaté (UNITAU). O projeto de pesquisa foi submetido e aprovado pelo Comitê de Ética em Pesquisa da UNITAU (protocolo $\mathrm{n}^{\circ}$. 353/03). A coleta foi realizada após leitura do termo de consentimento livre e esclarecido pelas pacientes que concordaram em participar da pesquisa.

Das 51 pacientes examinadas, 13 apresentaram quadro clínico e microbiológico sugestivo de CVV e 38 pacientes não apresentaram esses sinais. Os critérios de inclusão para o grupo de CVV foram presença de secreção esbranquiçada, placas brancas aderidas à mucosa, eritema e prurido e também exame microbiológico comprovando a presença de leveduras do gênero $C$ andida.

Foi coletada amostra de secreção vaginal utilizando swab estéril embebido em solução fisiológica $(\mathrm{NaCl}$ $0,85 \%$ ) por meio de movimentos circulares na região cérvico-vaginal. Após a coleta, o swab foi imerso em tubo contendo $2 \mathrm{~mL}$ de solução fisiológica, também estéril. A coleta deste material foi realizada pelo médico do Serviço de Ginecologia responsável pelo atendimento da paciente, após avaliação clínica.

Foram coletados $2 \mathrm{~mL}$ de saliva em coletor plástico universal estéril sem estímulo prévio. Uma parte das amostras coletadas foi utilizada para cultivo de Candida, e $1 \mathrm{~mL}$ foi armazenado em flaconetes contendo $20 \mu \mathrm{L}$ de fenilmetilsulfonil fluoride $\left(\operatorname{Sigma}^{\circledR}\right)$ para inibição de enzimas salivares e $20 \mu \mathrm{L}$ de azida sódica (Difco) e foram armazenados em freezer a $-20^{\circ} \mathrm{C}$ para posterior investigação de IgA. A seguir, o material coletado foi enviado ao Laboratório de Microbiologia do Instituto Básico de Biociências da UNITAU, respeitando-se período máximo de três horas entre coleta e processamento das amostras.

Alíquotas de $0,1 \mathrm{~mL}$ das amostras de saliva e de secreção vaginal puras e diluídas 1/10 e 1/100 foram semeadas, em duplicata, em ágar Sabouraud dextrose (DAS) acrescido de $0,1 \mathrm{mg} / \mathrm{mL}$ de meio de cultura de cloranfenicol (Quemicetina succinato, Carlo Erba ${ }^{\circledR}$ ). Após o crescimento das colônias características foram realizados esfregaços que foram corados pelo método de Gram e as mesmas colônias foram contadas nas placas que continham entre 30 e 300 colônias. Após cálculo das diluições, foi obtido o número de unidades formadoras de colônias por mililitro (ufc/mL). As colônias sugestivas foram repicadas em tubos contendo DAS para a obtenção de cultura pura. Posteriormente, as colônias foram identificadas por meio de provas morfológicas (formação de tubo germinativo em soro estéril de coelho e produção de clamidoconídeo em microcultivo em meio ágar-fubá Tween 80) e provas 
bioquímicas (fermentação e assimilação de carboidratos) de acordo com Sandven ${ }^{13}$.

Para dosagem de IgA salivar anti-Candida, foram sensibilizadas duas placas de microcultivo de plástico flexível e fundo chato (Hemobag ${ }^{\circledR}$ ) de 96 poços, com antígeno de parede de $C$. albicans preparado a partir de amostra padrão (ATCC- 18804) segundo Koga-Ito ${ }^{14}$. Este antígeno foi diluído em tampão carbonato-bicarbonato na concentração $15 \mathrm{mg} / \mathrm{mL}$. A seguir, as placas foram incubadas em estufa a $37{ }^{\circ} \mathrm{C}$ por duas horas e foram armazenadas a $4{ }^{\circ} \mathrm{C}$ por 48 horas. Após esse período, as placas foram lavadas com solução fisiológica tamponada com fosfato (PBS) e bloqueadas com $0,5 \%$ de gelatina em PBS, por 45 minutos a $37{ }^{\circ} \mathrm{C}$. Em seguida, foram lavadas cinco vezes com PBS 0,2\% Tween 20 (PBS-T). As amostras de saliva foram diluídas $(1 / 10 ; 1 / 20 ; 1 / 40)$ em PBS-T gelatina e foram colocados $100 \mu \mathrm{L}$ das diluições nos orifícios das placas, as quais foram incubadas a $37^{\circ} \mathrm{C}$ por duas horas e, logo após, lavadas com PBS-T. Os testes foram feitos em duplicatas. A seguir, foram adicionados $50 \mu \mathrm{L}$ do conjugado anti-IgA humana $\left(\operatorname{Sigma}^{\circledR}\right)$, marcado com peroxidase na concentração $1 / 1.000$, e as placas foram novamente incubadas a $37^{\circ} \mathrm{C}$ por uma hora e, em seguida, lavadas com PBS-T. Para verificar a reação, foi adicionado, em cada orifício, $100 \mu \mathrm{L}$ de reagente composto de $36 \mathrm{~mL}$ de tampão citrato ácido cítrico 0,1 M, $24 \mathrm{mg}$ de ortofenildiamina $\left(\right.$ Sigma ${ }^{\circledR}$ ) e $30 \mu \mathrm{L}$ de $\mathrm{H}_{2} \mathrm{O}_{2}$ a $30 \%$. A reação foi revelada e, após dez minutos, foi bloqueada com ácido sulfúrico $2,5 \mathrm{~N}$. As densidades ópticas (DO) foram lidas no leitor de Enzyme-Linked ImmunoSorbent Assay (ELISA, modelo 3550, BioRad $^{\circledR}$ ) com comprimento de onda de $490 \mathrm{~nm}$.

Os dados obtidos na análise de IgA salivar do Grupo Controle e do grupo de pacientes com candidose clínica foram avaliados e comparados por análise estatística, utilizando o teste $t$ de Student, (programa Epi-Info 2002), adotando-se o nível de significância $\mathrm{p}<0,05$.

\section{Resultados}

Das 51 mulheres avaliadas, $13(25,5 \%)$ apresentaram diagnóstico clínico de CVV, e destas, cinco (9,8\%) apresentaram leveduras do gênero Candida também na cavidade bucal. Não foi observada a presença do fungo em $23(45,1 \%)$ mulheres nos sítios mucosos estudados. Pôde-se observar também que, das 15 mulheres sem clínica de CVV, $11(73,3 \%)$ apresentaram a levedura na cavidade bucal, quatro $(26,7 \%)$ na mucosa vaginal e três (20\%) em ambos os sítios.

Foram isoladas 36 cepas de Candida nas mulheres avaliadas. A espécie prevalente foi $C$. albicans $(83,33 \%)$ em ambas as mucosas, tanto nas mulheres com CVV quanto nas que não apresentaram este quadro clínico. A
Tabela 1 - Médias e desvios padrão das densidades ópticas de lgA salivar em mulheres com ou sem leveduras do gênero Candida na cavidade bucal e vaginal

\begin{tabular}{l|r|c|c}
\hline Presença de Candida & n & Média & Desvio padrão \\
\hline Cavidade bucal & 8 & 0,674063 & 0,502411 \\
Cavidade vaginal & 12 & $0,373708^{*}$ & 0,273091 \\
Cavidade bucal e vaginal & 8 & $0,391250^{*}$ & 0,258353 \\
Ausência de Candida & 23 & 0,636326 & 0,452448 \\
\hline
\end{tabular}

*Diferença significante em relação ao Grupo Ausência de Candida.

Tabela 2 - Médias e desvios padrão das densidades ópticas de lgA salivar em mulheres com e sem candidíase vulvo-vaginal (CVV) e sua correlação com presença de leveduras do gênero Candida na vagina

\begin{tabular}{lccc}
\hline Candidíase vulvo-vaginal & n & Média & Desvio padrão \\
\hline Com CVV & 13 & 0,33 & 0,25 \\
Sem CVV e com Candida na vagina & 15 & $0,57^{*}$ & 0,41 \\
Sem CVV e sem Candida na vagina & 23 & $0,63^{*}$ & 0,45 \\
\hline
\end{tabular}

*Diferença significante em relação ao grupo com CVV.

outra espécie isolada, em menor proporção, foi Candida tropicalis $(16,66 \%)$, presente na vagina de quatro pacientes $(11,1 \%)$ e na cavidade bucal de uma $(2,8 \%)$ mulher com CVV. C. tropicalis foi isolada também da cavidade bucal de uma $(2,8 \%)$ paciente sem CVV.

Os dados obtidos por meio da DO para quantificação de anticorpos IgA anti-Candida revelaram que pacientes com diagnóstico clínico de CVV apresentaram níveis mais baixos de $\operatorname{IgA}$ na saliva $(\mathrm{DO}=0,34)$ quando comparados com os dados obtidos de pacientes sem CVV com presença de Candida na vagina ( $\mathrm{DO}=0,57)$, e pacientes sem CVV e sem Candida na vagina ( $D O=0,63)$; estas diferenças foram significativas (Tabelas 1 e 2).

No Grupo Controle, sete mulheres $(18,4 \%)$ apresentavam colonização por leveduras na mucosa vaginal e $11(21,6 \%)$, na cavidade bucal. No grupo com CVV, cinco mulheres $(9,8 \%)$ apresentaram leveduras do gênero Candida na saliva.

A média de ufc/mL de leveduras do gênero Candida no grupo com CVV, nas secreções salivares e vaginais, foram respectivamente de 6.030 e 52.723 (Tabela 3). No Grupo Controle, a média de ufc/mL encontrada na secreção vaginal foi de 1.973 e na saliva, 1.920 - sem diferença significante. No entanto, houve diferença significante quando se comparou a quantidade de Candida na vagina nos Grupos CVV e Controle.

\section{Discussão}

A presença de leveduras do gênero Candida no ambiente vaginal e também em outras mucosas, como a oral, em tão larga escala na população, permite classificar este microrganismo como saprófita, ou seja, a sua presença nestas mucosas não significa necessariamente doença. Entretanto, estudos demonstraram que $75 \%$ das 
Tabela 3 - Unidades formadoras de colônias de leveduras do gênero Candida coletadas da cavidade bucal e da vagina de mulheres com e sem candidiase vulvo-vaginal (CVV)

\begin{tabular}{|c|c|c|c|c|c|}
\hline \multirow{2}{*}{ Mulheres } & \multicolumn{2}{|c|}{ Com CVV (n=13) } & \multirow{2}{*}{ Mulheres } & \multicolumn{2}{|c|}{ Sem CVV (n=15) } \\
\hline & Candida na boca & Candida na vagina & & Candida na boca & Candida na vagina \\
\hline 1 & 23.050 & 92.750 & 1 & 490 & 8.625 \\
\hline 3 & 3.100 & 93.000 & 3 & 1.700 & - \\
\hline 4 & - & 201.500 & 4 & 7.250 & 83 \\
\hline 5 & 600 & 10.000 & 5 & 360 & - \\
\hline 7 & - & 45.500 & 7 & 120 & - \\
\hline 8 & - & 49.500 & 8 & 400 & - \\
\hline 9 & $\cdot$ & 39.500 & 9 & 2.350 & 1.275 \\
\hline 10 & 3.100 & 24.650 & 10 & 700 & - \\
\hline 11 & - & 13.600 & 11 & 1.400 & - \\
\hline Média+dp & $6.030 \pm 9.607$ & $52.942 \pm 52.723^{*}$ & Média $\pm d p$ & $1.920 \pm 2.120$ & $1.973 \pm 2.993$ \\
\hline
\end{tabular}

*Diferença significativa em relação à presença de Candida na vagina das mulheres sem CVV.

$d p=$ desvio padrão

mulheres assintomáticas com colonização por Candida, em algum momento, deverão apresentar algum episódio de infecção clínica no decorrer de sua vida, e $40 \%$ terão um segundo episódio ${ }^{15}$.

No presente trabalho, observou-se que $18,4 \%$ das pacientes do Grupo Controle (assintomáticas) apresentaram Candida na mucosa vaginal, com taxas de ufc/mL menores quando comparadas ao grupo com CVV, demonstrando a colonização desta mucosa por esse fungo e a sua participação na microbiota residente, sem entretanto causar dano. Os dados obtidos estão de acordo com outro estudo, no qual se demonstrou cultura positiva para C. albicans em mulheres saudáveis.

Diferentes espécies de cultura para leveduras do gênero Candida apresentam potencial patogênico para seres humanos e animais, mas a espécie $C$. albicans apresenta maior variedade de fatores de virulência, sendo prevalente nas mucosas, com médias de 50 a $80 \%$ do total de cepas isoladas, sendo, portanto, considerada a espécie mais patogênica ${ }^{3,6,7,16,17}$.

C. albicans foi a espécie mais prevalente em ambos os grupos do novo estudo, tanto na secreção vaginal quanto na saliva, concordando com dados da literatura que demonstraram que, dentre os diversos fatores que influenciam a prevalência de $C$. albicans nas mucosas, destacam-se a sua maior habilidade e a capacidade de aderência a vários receptores celulares ${ }^{18,19}$. Entretanto, a aderência, fase essencial para a levedura progredir no processo de colonização e infecção, pode ser impedida ou diminuída pelas defesas dos organismos na mucosa, por intervenção das IgAs que promovem a neutralização do fungo pela ligação em suas adesinas, bloqueando, conseqüentemente, sua ligação a receptores celulares ${ }^{20}$.
A IgAs protege a mucosa do contato com o microrganismo antes de sua penetração, formando complexo Ag-Ac, que é removido pela secreção de mucina proveniente de tecidos glandulares localizados em vários sítios da mu$\operatorname{cosa}^{21}$. Demonstrou-se que existe uma resposta local com produção de IgA anti-Candida em pacientes com cultura positiva para a levedura ${ }^{22}$, sugerindo que a síntese de anticorpos associada às mucosas estaria sendo estimulada na vigência de uma maior quantidade de leveduras ou de infecção ativa, ao passo que a resposta sistêmica pode refletir uma exposição anterior e está mais acentuada em pacientes com cultura negativa.

Gregory e Allansmith ${ }^{23}$ demonstraram que a presença de antígenos em determinados sítios induzem grande expressão clonal, que resulta em níveis elevados de anticorpos IgA no local e em outros sítios não expostos ao antígeno simultaneamente. Este fato não foi observado no presente estudo, no qual mulheres com CVV apresentaram maior quantidade de leveduras do gênero Candida, tanto na cavidade vaginal quanto na saliva, e menores concentrações de $\operatorname{Ig} A$, demonstrando o importante papel deste anticorpo no equilíbrio da relação parasita-hospedeiro, pois sua diminuição provavelmente favoreceu a proliferação do microrganismo presente, passando do estágio de colonização à infecção.

Em contrapartida, nas pacientes do Grupo Controle, as médias de $\mathrm{ufc} / \mathrm{mL}$ de leveduras do gênero Candida, tanto na saliva quanto na secreção vaginal, foram mais baixas, e a concentração de $\operatorname{IgA}$ salivar mostrou-se mais elevada - o que provavelmente conferiu proteção nestes sítios, impedindo a adesão das células leveduriformes à mucosa. 
Pacientes com papilomavírus humanos (HPV) genital apresentaram níveis mais baixos de $\operatorname{Ig}$ A salivar quando comparados aos níveis de $\operatorname{Ig} A$ do grupo de pacientes sem $\mathrm{HPV}^{24}$, sugerindo que a baixa produção destes anticorpos nas mucosas predispõe à implantação de patógenos.

Demonstrou-se também que IgAs anti-Candida da saliva e do leite bovino promoveram a inibição da aderência deste microrganismo em cultura de células epiteliais ${ }^{25}$. Estes autores observaram também que o componente secretor desta imunoglobulina pode se ligar a $C$. albicans de forma não imune, ajudando no processo de inibição da aderência.

Apesar de a imunidade adquirida mediada por células com elevada síntese de IL-2 durante o início da infecção ser essencial para eliminação de Candida das mucosas do hospedeiro ${ }^{26}$, o aumento do nível de anticorpos nas mucosas aumenta a velocidade de remoção do fungo, ajudando na proteção local. A IgAs tem sido implicada na inibição de microrganismos das mucosas, removendo-os desses sítios pela formação de imunocomplexos ${ }^{23,24,27}$.

McArthur e Clark ${ }^{28}$ demonstraram que anticorpos IgA anti-Candida produzidos localmente são distribuídos para diferentes locais do organismo pela circulação sanguínea, conferindo proteção em outras mucosas.

A participação de imunidade celular nas vaginites fúngicas é o processo eficaz na eliminação do fungo. No entanto, a imunidade humoral tem papel fundamental, protegendo a mucosa por meio da IgAs, inibindo a etapa inicial no processo de implantação do fungo e, conseqüentemente, a infecção, como sugerem os resultados do presente trabalho, pois mulheres que apresentaram níveis mais altos de IgAs tiveram menor quantidade de leveduras em ambas as mucosas - vaginal e bucal.

\section{Referências}

1. Williams DW, Potts AJ, Wilson M, Matthews JB, Lewis MA. Characterisation of the inflammatory cell infiltrate in chronic hyperplastic candidosis of the oral mucosa. J Oral Pathol Med. 1997;26(2):83-9.

2. Sherman RG, Prusinki L, Ravenel MC, Joralmon RA. Oral candidosis. Quintessense Int. 2002;33(7):521-32.

3. Val ICC, Almeida Filho GL. Abordagem atual da candidíase vulvovaginal. DST J Bras Doenças Sex Transm. $2001 ; 13(4): 3-5$.

4. Oliveira JM, Cruz AS, Fonseca AF, Vaz CP, Rodrigues A, Aurea F, et al. Prevalence of Candida albicans in vaginal fluid of asymptomatic Portuguese women. J Reprod Med. 1993;38(1):41-2.

5. Takakura N, Sato $Y$, Ishibashi $H$, Oshima H, Uchida K, Yamaguchi $\mathrm{H}$, et al. A novel murine model of oral candidiasis with local symptoms characteristic of oral thrush. Microbiol Immunol. 2003;47(5):321-6.

6. Cordeiro SN, Turato ER, Vicentini RMR, Gonçalves AKS, Giraldo PC. Hábitos de higiene e sexuais de mulheres com vulvovaginites recorrentes. DST J Bras Doenças Sex Transm. 2003;15(2):15-9.

7. Bastos AMC, Bravo RS, Goulart Filho RA, Isalan TB, Barreto NA. Perfil das mulheres com processo inflamatório por Candida em resultados de colpocitologia oncológica numa clínica de DST. DST J Bras Doenças Sex Transm. 2003;15(2):26-38.

8. Giraldo PC. A imunologia em vulvovaginites de repetição. DST J Bras Doenças Sex Transm. 2002;14(3):30.

9. Jeganathan S, Chan YC. Imunodiagnosis in oral candidiasis. A review. Oral Surg Oral Med Oral Pathol. 1992;74(4):451-4.

10. Badauy CM, Barbachan JJ, Rados PV, Sant'ana Filho M, Chies JA. Relationship between Candida infection and immune cellular response in inflammatory hyperplasia. Oral Microbiol Immunol. 2005;20(2):89-92.
11. Giraldo PC, Gonçalves AK, Eleutério Junior J. Secretory immunoglobulin A: a protective factor in the genital mucosa. Braz J Infect Dis. 2006; 10(4):232-4.

12. Polonelli L, Conti S, Menozzi MG, Gerloni M, Elosegui R, Fernandez $M$, et al. Diagnostic potential of IgA coated Candida cells in mucous membrane candidiasis. Mycopathologia. 1991; 1 16(2):105-12.

13. Sandven P. Laboratory identification and sensitivity testing of yeast isolates. Acta Odontol Scand. 1990;48(1):27-36.

14. Koga-lto CY. Correlação entre a presença de Streptococcus do grupo mutans e Candida com níveis de lgA na saliva humana [tese]. Campinas (SP): Unicamp; 1997.

15. Mårdh PA, Rodrigues AG, Genç M, Novikova N, Martinez-deOliveira J, Guaschino S. Facts and myths on recurrent vulvovaginal candidosis-a review on epidemiology, clinical manifestations, diagnosis, pathogenesis and therapy. Int J STD AIDS. 2002; 13(8):522-39.

16. Ferraza MHSH, Maluf MLF, Consolaro MEL, Shinobu CS, Svidzinski TIE, Batista MR. Caracterização de leveduras isoladas da vagina e sua associação com candidíase vulvovaginal em duas cidades do sul do Brasil. Rev Bras Ginecol Obstet. $2005 ; 27(2): 58-63$.

17. Galle LC, Gianinni MSM. Prevalência e susceptibilidade de leveduras vaginais. J Bras Patol Med Lab. 2004;40(4):229-36.

18. Calderone RA, Fonzi WA. Virulence factors of Candida albicans. Trends Microbiol. $2001 ; 9(7): 327-35$.

19. Cotter G, Kavanagh K. Adherence mechanisms of Candida albicans. Br J Biomed Sci. 2000;57(3):241-9.

20. van Egmond $M$, Damen $C A$, van Spriel $A B$, Vidarsson $G$, van Garderen $E$, van de Winkel JG. $\lg A$ and $\lg A$ Fc receptor. Trends Immunol. 2001;22(4):205-11. 
21. Russell MW, Mestecky J. Humoral immune responses to microbial infections in the genital tract. Microbes Infect. 2002;4(6): 667-77.

22. Carvalho RJV, Cunha CM, Silva DAO, Sopelete MC, Urzedo JE, Moreira TA, et al. $\lg A, \lg E$, e subclasses de $\lg G$ anti-Candida albicans no soro e lavado vaginal de pacientes com candidíase vulvovaginal. Rev Assoc Med Bras. 2003;49(4):434-8.

23. Gregory RL, Allansmith MR. Naturally occurring IgA antibodies to ocular and oral microorganisms in tears saliva and colostrum: evidence for a common mucosal immune system and local immune response. Exp Eye Res. 1986;43(5):739-49.

24. Gonçalves AK, Giraldo P, Barros-Mazon S, Gondo ML, Amaral RL, Jacyntho C. Secretory immunoglobulin A in saliva of women with oral and genital HPV infection. Eur J Obstet Gynecol Reprod Biol. 2006; 124(2):227-31.

25. Holmes AR, Bandara BM, Cannon RD. Saliva promotes Candida albicans adherence to human epithelial cells. J Dent Res. 2002;81(1):28-32.

26. Ouyang W, Chen S, Liu Z, Wu Y, Li J. Local Th1/Th2 cytokine expression in experimental murine vaginal candidiasis. J Huazhong Univ Sci Technolog Med Sci. 2008;28(3):352-5.

27. Fidel PL Jr. Immunity in vaginal candidosis. Curr Opin Infect Dis. 2005; 18(2):107-11.

28. McArthur WP, Clark WB. Specific antibodies and their potential role in periodontal diseases. J Periodontol. 1993;64/8 Suppl):807-18. 